\title{
Enhanced Accuracy in Novel Mirror Drawing after Repetitive Transcranial Magnetic Stimulation-Induced Proprioceptive Deafferentation
}

\author{
Daniela Balslev, ${ }^{1,3}$ Lars 0. D. Christensen, ${ }^{4}$ Ji-Hang Lee, ${ }^{4}$ Ian Law, ${ }^{1,2}$ Olaf B. Paulson, ${ }^{1,3}$ and R. Christopher Miall ${ }^{4}$ \\ ${ }^{1}$ Neurobiology Research Unit and ${ }^{2}$ Department of Clinical Physiology and Nuclear Medicine, Rigshospitalet, Copenhagen University Hospital, DK-2100 \\ Copenhagen, Denmark, ${ }^{3}$ Danish Research Centre for Magnetic Resonance, Hvidovre Hospital, 2650 Hvidovre, Denmark, and ${ }^{4}$ University Laboratory of \\ Physiology, University of Oxford, Oxford OX1 3PT, United Kingdom
}

\begin{abstract}
When performing visually guided actions under conditions of perturbed visual feedback, e.g., in a mirror or a video camera, there is a spatial conflict between visual and proprioceptive information. Recent studies have shown that subjects without proprioception avoid this conflict and show a performance benefit. In this study, we tested whether deafferentation induced by repetitive transcranial magnetic stimulation (rTMS) can improve mirror tracing skills in normal subjects. Hand trajectory error during novel mirror drawing was compared across two groups of subjects that received either $1 \mathrm{~Hz}$ rTMS over the somatosensory cortex contralateral to the hand or sham stimulation. Mirror tracing was more accurate after rTMS than after sham stimulation. Using a position-matching task, we confirmed that rTMS reduced proprioceptive acuity and that this reduction was largest when the coil was placed at an anterior parietal site. It is thus possible, with rTMS, to enhance motor performance in tasks involving a visuoproprioceptive conflict, presumably by reducing the excitability of somatosensory cortical areas that contribute to the sense of hand position.
\end{abstract}

Key words: proprioceptive; visual; somatosensory; hand; parietal; motor activity

\section{Introduction}

Exposure to a novel visuomotor spatial transformation results in an immediate decrease in movement accuracy (Ghez et al., 2000). This problem arises when movements are visually monitored in a mirror or a video display and the performer has insufficient experience with the transformation from the hand to the visual space. In real life, for instance, it can happen to a surgeon who adjusts the view direction of the laparoscope to get a better view of the operation field or to a dentist who needs a mirror to work on the backside of the tooth.

Intriguingly, performance deteriorates less in proprioceptively deafferented patients than in healthy controls when they change from normal to mirror drawing. In the absence of proprioception, it is easier to maintain movement speed and accuracy when the new mapping from the hand to the visual space is introduced (Lajoie et al., 1992; Guedon et al., 1998). Furthermore, in healthy persons who are adapting to a visuomotor transformation, movement accuracy correlates negatively with the firing activity of the muscle spindles (Jones et al., 2001). These findings lead to the hypothesis that reducing proprioceptive inflow to the

Received April 29, 2004; revised Aug. 25, 2004; accepted Sept. 4, 2004.

This work was supported by the Wellcome Trust, the James S. McDonnell Foundation, Copenhagen University Hospital, and the Foundation for Research in Neurology.

Correspondence should be addressed to Dr. Daniela Balslev, Neurobiology Research Unit, N9201, Copenhagen University Hospital, Rigshospitalet, Blegdamsvej 9, DK-2100 Copenhagen, Denmark. E-mail: daniela@nru.dk. DOI:10.1523/JNEUROSCI.1738-04.2004

Copyright $\odot 2004$ Society for Neuroscience $\quad$ 0270-6474/04/249698-05\$15.00/0 brain improves performance in situations with a visuoproprioceptive conflict.

To test this hypothesis, it is necessary to reduce proprioceptive accuracy in healthy people. To this end, we applied 15 min of $1 \mathrm{~Hz}$ repetitive transcranial magnetic stimulation (rTMS) over the anterior parietal cortex, at the putative location of the hand area in the primary somatosensory cortex. rTMS at $1 \mathrm{~Hz}$ reduces cortical excitability, producing a so-called "virtual lesion" (PascualLeone et al., 1999; Siebner and Rothwell, 2003). In neurological patients, a lesion of the anterior parietal cortex impairs the sense of position of the contralateral arm (Corkin et al., 1970) but spares the ability to perform voluntary movements (Pause et al., 1989). Here, we show that rTMS applied at an anterior parietal site improved trajectory accuracy during a novel mirror drawing task performed with the contralateral hand. In two separate control experiments, we confirmed that rTMS decreased proprioceptive accuracy and that this effect could be located in the anterior parietal lobe.

\section{Materials and Methods}

Experiment 1: mirror tracing

Subjects. Twelve healthy, right-handed volunteers (six females, 18-42 years of age, median 28) gave written informed consent and participated in the experiment. All of the subjects had normal or corrected-to-normal vision and used a computer mouse daily. The study was approved by the Central Oxfordshire Research Ethics Committee.

Study design. Each subject was randomly assigned to an intervention group, either real-rTMS or sham-rTMS stimulation. There was no significant difference between groups in median age or female/male ratio.

Task. The task was a computer implementation of the classic mirror 


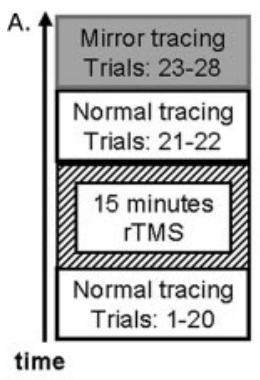

B.

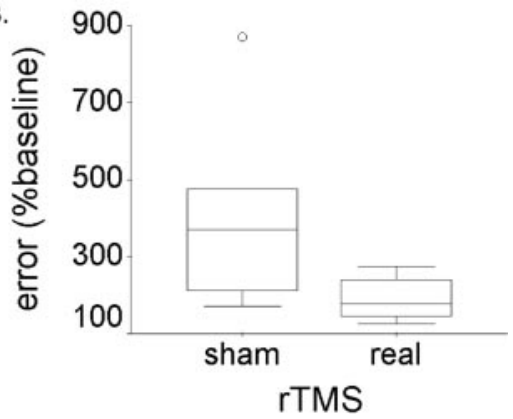

Figure 1. The mirror tracing experiment. $A$, Study design. Normal tracing skill was tested before and immediately after $15 \mathrm{~min}$ of $1 \mathrm{~Hz}$ rTMS. Mirror tracing was tested during six trials given after rTMS. Each trial lasted $10 \mathrm{sec}$ with an intertrial interval of $10 \mathrm{sec}$. B, Results. Box plot of trajectory error during novel mirror tracing in the groups that received either real or sham rTMS. Post-rTMS error is expressed as percentage of the baseline error sampled before rTMS. Mann-Whitney $U$ test, $p<0.05 ; n=6$ subjects in each group.

drawing task (Milner et al., 1968) on which patients without proprioception outperform healthy controls (Lajoie et al., 1992). Trials of normal tracing were performed before (20 trials) and after (two trials) rTMS, followed immediately by six trials of mirror tracing (Fig. $1 A$ ).

The subjects sat comfortably at a table, with the chin on a support that aligned the head to the body midline. A computer screen $(330 \times 240 \mathrm{~mm}$, $640 \times 480$ pixels) was placed at $56 \mathrm{~cm}$ in front of them. With the right index finger, the subjects operated a sliding computer "mouse" (FELIX Pointing Device; Altra, Rawlins, WY), which moved in an active area of $30 \times 24 \mathrm{~mm}$. The mouse controlled a screen cursor (radius, $2 \mathrm{~mm}$; visual angle, $0.42^{\circ}$ ). The subjects were instructed to move the cursor in a clockwise direction along a circular path (radius, $90 \mathrm{~mm}$; visual angle, $18.82^{\circ}$; path width, $10 \mathrm{~mm}$ ) and to choose the highest possible speed of tracing that allowed them to stay within the path. Each trial lasted $10 \mathrm{sec}$. Between trials, the subjects had $10 \mathrm{sec}$ to place the cursor in the start position marked at the leftmost point of the circular path and to prepare for a new trial. During normal tracing trials and all of the intertrial intervals, the trajectory of the cursor was similar to the trajectory of the mouse $\left(x_{\text {cursor }}=x_{\text {mouse }} ; y_{\text {cursor }}=y_{\text {mouse })}\right.$. During the mirror tracing trials, the position of the cursor was up-down reversed relative to the position of the mouse $\left(x_{\text {cursor }}=x_{\text {mouse }} ; y_{\text {cursor }}=-y_{\text {mouse }}\right)$. A laptop running E-prime (Psychology Software Tools, Pittsburgh, PA) on Windows 2000 presented the stimuli and recorded the position of the mouse and cursor every $50 \mathrm{msec}$.

rTMS. Each rTMS session consisted of 900 biphasic stimuli produced by a Magstim Rapid magnetic stimulator (The Magstim Company, Whitland, UK) and delivered with a frequency of $1 \mathrm{~Hz}$ over 15 min.

One of two identical, standard 70-mm-diameter figure-of-eight coils was centered over the stimulation site and maintained in this position by a coil holder. This site was mapped in each subject in relation to the "motor hotspot" of the left hemisphere, which is the scalp projection of the primary motor cortex (Wassermann et al., 1996). The site of stimulation was located at $3 \mathrm{~cm}$ posterior to the motor hotspot, measured on a line oriented at $45^{\circ}$ from the sagittal plane and perpendicular on the central sulcus. Previous TMS studies that successfully targeted the somatosensory hand area report a coil position at $1-4 \mathrm{~cm}$ posterior to the motor hotspot (Sugishita and Takayama, 1993; Harris et al., 2002; McKay et al., 2003; Ragert et al., 2004). Thus, we assumed that positioning the coil at $3 \mathrm{~cm}$ from the motor hotspot would reduce the activity of the primary somatosensory cortex with minimum effect on the primary motor cortex. The motor hotspot was defined as the point of maximum evoked motor response in the relaxed first dorsal interosseus (FDI) muscle of the right hand. Stimulation intensity was set at $110 \%$ of resting motor threshold of the right FDI muscle. To identify the resting motor threshold, the subjects were asked to rest the right hand on the table with the fingers slightly spread. The resting motor threshold was defined as the lowest intensity that reliably elicited a visible twitch in the FDI muscle when the stimulation was given over the motor hotspot. During real
rTMS, the coil was positioned tangential to the scalp with the long axis of the figure-of-eight coil oriented at $45^{\circ}$ to the parasagittal plane. The current flow of the initial rising phase of the biphasic pulse in the TMS coil induced a current flowing from posterior to anterior in the brain. During sham rTMS, the coil was tilted at $90^{\circ}$ to the scalp, with one wing of the coil in contact with the scalp over the site of stimulation. This coil arrangement reproduces the acoustic sensation of real rTMS, with minimal effects on the cortex (Lisanby et al., 2001). During each session, the active coil was exchanged for the spare coil after exactly 4 and 11 min of rTMS to avoid overheating. All of the subjects were tested on the mirror tracing task (experiment 1 ) within 3 min after the cessation of the rTMS train and on the finger-to-finger matching task (experiments 2 and 3 ) within 5 min after the last rTMS pulse.

Data analysis. Motor performance during each trial was quantified by the speed of movement (calculated as the ratio between total distance covered by the cursor and movement time) and by the modulus of the radial trajectory error (Poulton, 1974). Radial trajectory error was calculated as the difference between the distance from the center of the circular path to the cursor and radius of the circular path. For each $10 \mathrm{sec}$ trial, the absolute radial trajectory error was averaged over measurements taken at $20 \mathrm{~Hz}$. For each subject, the measures of performance were averaged over baseline, tracing, and mirror tracing trials, respectively. Post-rTMS performance was expressed as a percentage of the subject's pre-TMS baseline performance. Data were inspected for normality and outliers using box plots. The effect of rTMS on performance was tested across groups, using the Mann-Whitney $U$ test as implemented in SPSS version 11.5 (SPSS, Chicago, IL). Furthermore, the effect of rTMS on rate of learning during mirror tracing was investigated by comparing between groups the slope of the regression line through error values over the six mirror tracing trials.

\section{Experiment 2: finger matching}

Subjects. Ten subjects (three females, $18-48$ years of age, median 29) participated in this experiment.

Study design. Each subject participated in two experimental sessions, real rTMS and sham rTMS, scheduled on 2 separate days, $2 \mathrm{~d}$ apart. The order of session presentation was randomized and counterbalanced across subjects. During each session, proprioception was tested twice, before and after rTMS.

Task. Proprioception was tested using a "matching" paradigm (Paillard and Brouchon, 1968; Velay et al., 1989). Without seeing their hands, the subjects matched with their left index finger the position of their right index finger that was passively moved by the examiner.

The apparatus consisted of two LEGO bricks (LEGO Company, Billund, Denmark) (Fig. 2) (length, $125 \mathrm{~mm} ; 16$ cylindrical protrusions or "studs"; distance between studs, $8 \mathrm{~mm}$ ) fixated at $56 \mathrm{~mm}$ from each other and placed on the table parallel with and symmetrical about the subjects' midsagittal plane. The subjects sat comfortably in front of the table with the chin on a support that aligned the head to the body midline, the elbows were flexed, and the forearms were placed on the table symmetrically about the midsagittal plane. They were instructed to keep their eyes closed during the test. At the beginning of each trial, the subjects placed their index fingers on the studs located at the proximal end of both bricks. The examiner then lifted the subject's right index finger from this start position and placed it on a target stud located along the right brick, at variable distance from start. Then, the subject lifted their left index finger and put it down on the left brick on a stud that best matched the felt position of the right finger. Subjects were allowed to correct the position of their indicator finger until they were satisfied with the position match. The proprioceptive error was recorded as the number of studs by which the indicator finger missed the position of the target finger. This error was transformed in metric units using the following transformation: one interstud interval $=8 \mathrm{~mm}$. Finally, the examiner moved the subject's index fingers back to the start position to prepare for a new trial. No feedback on performance accuracy was provided. Each test consisted of 18 trials and took $<5 \mathrm{~min}$ to complete. The target positions were located at $8,16,24,32,40$, and $48 \mathrm{~mm}$ from start. Each target position was presented three times in a predetermined random order. The order of 


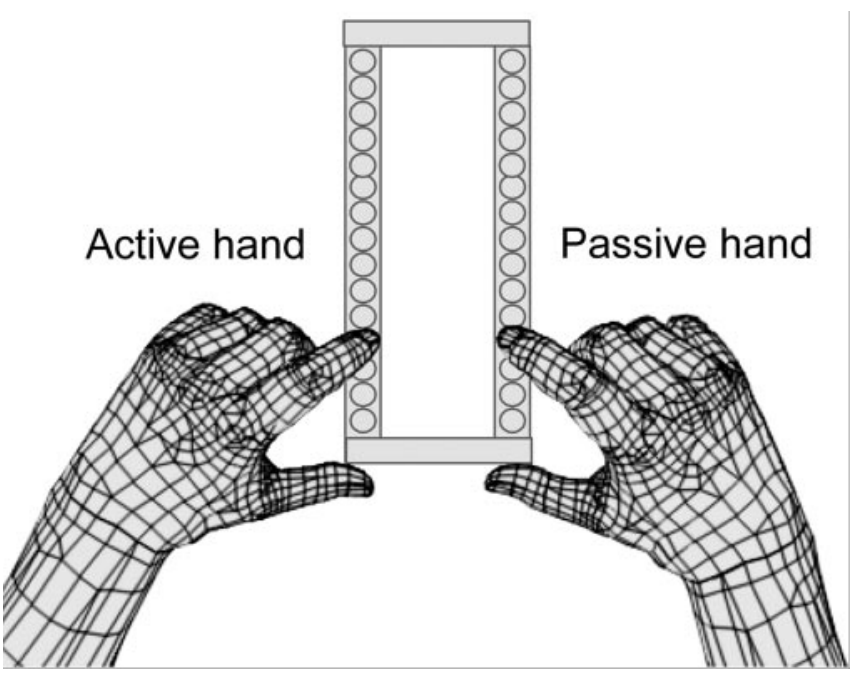

Figure 2. The apparatus used to test proprioceptive accuracy. The apparatus consisted of two LEGO bricks fixated on a horizontal table $s 0$ as to be parallel with the subject's midsagittal plane. Before each trial, the examiner positioned both fingers on the studs situated at the proximal end of the apparatus. Then, the subject's right index finger was passively moved to one of the studs on the right brick, at variable distance from the start position. Finally, the subject moved the left index finger to place it on a stud of the left brick so as to match the position of the right index finger. The subjects had their eyes closed throughout the experiment and received no feedback on performance.

target presentation was constant between subjects but differed within subject between tests.

Before the experiment, the subjects received three practice trials to become familiar with the task. The practice trials followed the same procedure, except that the subjects were allowed to keep their eyes open.

$r$ TMS. rTMS sessions followed the same protocol as for experiment 1.

Data analysis. For each subject, proprioceptive error was averaged across trials and expressed as a percentage from the subject's baseline (pre-TMS) error. Data was inspected for normality and outliers using box plots. The difference in accuracy after rTMS between the two intervention groups was tested using a paired-sample $t$ test, implemented in SPSS version 11.5.

Experiment 3: finger matching (variable location of rTMS coil) Subjects and task. Forty-five healthy, right-handed volunteers participated in the experiment. Each subject was assigned to one of three intervention groups that differed by the scalp location at which rTMS was applied: (1) primary motor cortex (M1) at the motor hotspot, (2) anterior parietal cortex (APC), $3 \mathrm{~cm}$ posterior to the motor hotspot, the same site as that used for experiments 1 and 2, and (3) posterior parietal cortex (PPC), $6 \mathrm{~cm}$ posterior to the motor hotspot. These distances were measured on a line oriented at $45^{\circ}$ from the sagittal plane, perpendicular to the central sulcus. Each group consisted of 15 subjects, aged between 19 and 42 years (median 24; M1 group), 18 and 48 years (median 29; APC group), and 19 and 33 years (median 26; PPC group). The subjects completed the finger-to-finger matching task from experiment 2 before and after rTMS. The rTMS protocol was the same as that used for experiments 1 and 2 .

In all of the experiments in this study, the statistical analysis was done on performance data that were normalized to the subject's pre-TMS performance and not on absolute post-rTMS performance measures. The reason for performing the statistical analysis on normalized data was to eliminate sources of nuisance variation between subjects. Subjectspecific factors, such as attention or motivation, are likely to influence motor performance and are difficult to control experimentally. Individual differences are especially important when proprioception is assessed using a position-matching test. For instance, the ability of the subject to reproduce the position of a finger that is moved passively to some target position depends on the location of this target within the range of finger motion allowed by the subject's hand anatomy (Janwantanakul et al.,

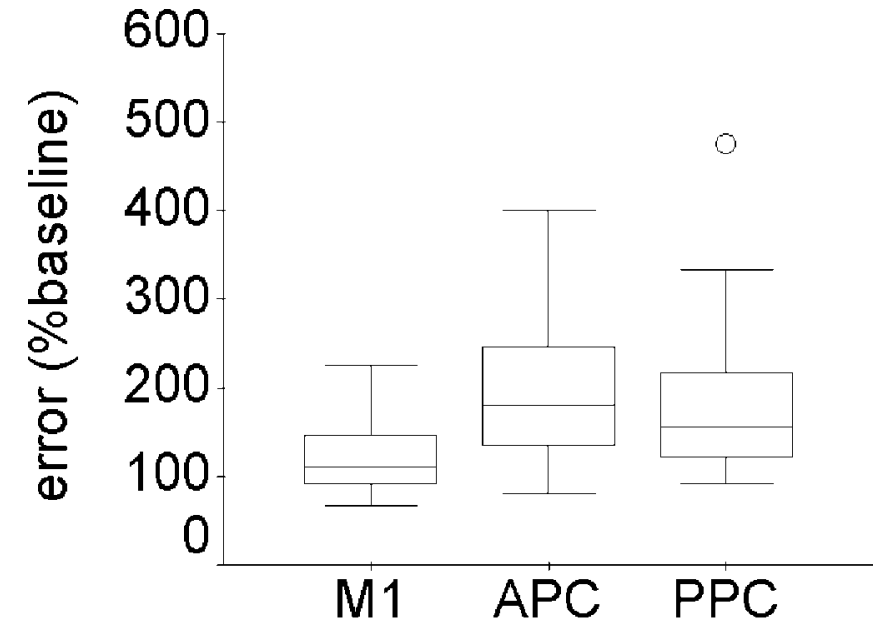

\section{Brain site}

Figure 3. Box plot of proprioceptive error after rTMS applied at various scalp sites. The coil was placed over three different brain sites: $M 1$, at the motor hotspot; $\mathrm{APC}$, at $3 \mathrm{~cm}$ posterior to the motor hotspot; and PPC, at $6 \mathrm{~cm}$ posterior to the motor hotspot. The effect of rTMS site on proprioceptive error was significant (Kruskal-Wallis test, $p=0.023$ ). Post-rTMS error is expressed as percentage of the baseline error, sampled before rTMS. $n=15$ subjects in each group.

2001) or on the ability of the subject to relax the finger during passive movement (Rymer and D'Almeida, 1980). We reasoned that subjectspecific effects were likely to affect both pre-TMS and post-TMS performance, so normalizing post-rTMS error relative to baseline would reduce noise and increase statistical power in this relatively small data sample.

\section{Results}

\section{Experiment 1}

Trajectory error during mirror tracing trials was significantly higher after sham than after real rTMS (Fig. $1 B$ ) (Mann-Whitney $U$ test, $U=5 ; p=0.037)$. The absolute mirror tracing error in the real-rTMS group was median $5.69 \mathrm{~mm}$, range $3.86-8.09 \mathrm{~mm}$ and in the sham group was median $8.81 \mathrm{~mm}$, range $4.18-22.24 \mathrm{~mm}$.

No difference was found across intervention groups in the speed of mirror tracing $(p=0.180)$, the speed of post-rTMS tracing in the normal task $(p=0.937)$, the error of post-rTMS normal tracing ( $p=0.485$ ), or learning speed (the slope of the regression of error score against trial number for the six mirror tracing trials; $p=0.485$ ). Thus, rTMS over the somatosensory cortex contralateral to the hand improved performance in the mirror tracing task compared with the sham stimulation.

\section{Experiment 2}

rTMS over the somatosensory cortex contralateral to the hand reduced proprioception compared with sham stimulation. After real-rTMS, proprioceptive error increased to $192.14 \pm 31.67 \%$ (mean \pm SE) of pre-TMS performance, whereas after sham, proprioceptive error was $95.68 \pm 15.37 \%$. The difference among the groups was significant (paired $t$ test, $p=0.043$ ). The absolute value for proprioceptive error after rTMS was median $4.22 \mathrm{~mm}$, range $1.78-7.56 \mathrm{~mm}$ for real-rTMS and median 3.33, range 1.33$6.67 \mathrm{~mm}$ for sham.

\section{Experiment 3}

There was a significant difference among the three groups in their proprioceptive matching accuracy after rTMS (Fig. 3), with the 
greatest reduction in proprioception for the group stimulated over the APC. The effect of rTMS site on proprioceptive accuracy was significant (Kruskal-Wallis test, $\chi^{2}=7.51 ; p=0.023$ ). In pairwise comparison among the three groups, the difference between APC and M1 (Mann-Whitney $U$ test, $U=52 ; p=0.012$ ) and the difference between PPC and M1 (Mann-Whitney $U$ test, $U=61 ; p=0.033$ ) were significant, whereas the difference between stimulation over APC and PPC was not significant $(U=$ 99.5; $p=0.58$ ). The absolute post-rTMS proprioceptive error was as follows: M1 group, median $4.89 \mathrm{~mm}$, range $1.78-12 \mathrm{~mm}$; APC group, median $4.89 \mathrm{~mm}$, range $1.78-9.33 \mathrm{~mm}$; and PPC group, median $5.77 \mathrm{~mm}$, range $3.11-8.44 \mathrm{~mm}$.

\section{Discussion}

rTMS applied to the anterior parietal cortex improved trajectory accuracy during the novel task of mirror tracing (experiment 1). A control experiment confirmed that the rTMS intervention reduced hand proprioception (experiment 2). These results support the hypothesis that motor control in situations with a visuoproprioceptive conflict benefits from a reduction in proprioception. We have put forth this hypothesis in the light of previous studies that report advantages in motor performance when the proprioceptive input is low (Jones et al., 2001) or absent (Lajoie et al., 1992; Guedon et al., 1998).

The rTMS protocol used in this experiment is known to produce a decrease in neural activity that lasts several minutes after the cessation of the stimulation pulses. This effect has been demonstrated neurophysiologically in the motor cortex (Chen et al., 1997) and confirmed behaviorally for various cortical areas, including the sensory cortices (Kosslyn et al., 1999; Boroojerdi et al., 2000; Knecht et al., 2003). For instance, 10-20 min of rTMS at $1 \mathrm{~Hz}$ and $110 \%$ motor threshold applied over the somatosensory cortex increases the threshold for discriminating tactile stimuli for a period of 4-8 min post-rTMS (Knecht et al., 2003). To the best of our knowledge, no study has investigated previously whether low-frequency rTMS decreases proprioceptive ability.

There are at least three possible brain regions involved in proprioceptive processing whose inactivation may have caused the decrease in the sense of finger position that followed rTMS stimulation $3 \mathrm{~cm}$ posterior to the motor hotspot. First, the primary somatosensory cortex is the first cortical station for proprioceptive afferents and its lesion impairs all of the somatosensory modalities (Corkin et al., 1970). Second, the motor area has neurons that respond to passive movement (Strick and Preston, 1982), and evidence has been put forth recently for its importance in somatosensation (Nudo et al., 2000; Naito et al., 2002). A third candidate area is the left posterior parietal cortex, whose lesion in neurological patients can cause deficits in locating body parts (Wolpert et al., 1998; Semenza, 2001). To find out which brain region was most likely to be responsible for the decrease in proprioception in this study, we compared position-matching error after rTMS at the motor hotspot and the anterior and posterior parietal cortex (experiment 3). The effect of rTMS on proprioceptive accuracy was maximal $3 \mathrm{~cm}$ posterior to the motor cortex. It decreased significantly when the coil was placed directly over the motor cortex at the motor hotspot. The size of the effect also decreased when the coil was moved $3 \mathrm{~cm}$ further posterior, although this difference was not statistically significant. Thus, the brain area responsible for the rTMS-induced decrease in proprioception is located anterior in the parietal lobe, probably corresponding to the representation of the hand in the somatosensory cortex. Additional experiments combining rTMS and neuroim- aging techniques may identify more directly the brain area that is responsible for this effect.

Patients with a decrease in proprioception have great difficulties with controlling their hand (Rothwell et al., 1982; Jeannerod et al., 1984; Sanes et al., 1985; Pause et al., 1989). Visual feedback, however, improves the accuracy of their movements (Ghez et al., 1995), reducing (Sanes et al., 1984) or even eliminating (Bard et al., 1995) the difference in performance between patients and healthy controls. Furthermore, in novel situations with optical displacement, proprioceptively deafferented patients are less impaired compared with healthy controls (Lajoie et al., 1992; Guedon et al., 1998). These observations in patients demonstrate that, when visual feedback is available, proprioception is less important and may even be an obstacle for adapting to visual perturbations. Because patients who have lost proprioception are usually included in experiments many years after the onset of deafferentation, it is difficult to tell whether their improved performance follows directly from the lack of proprioception or is merely a side effect reflecting, for instance, their familiarity with motor learning situations or their use of compensatory strategies. The present study demonstrates a performance benefit in healthy persons after proprioceptive deafferentation by rTMS over the anterior parietal cortex. This finding confirms that the cortical processing of proprioceptive inflow has a limited contribution to trajectory control during adaptation to visual perturbations. It also suggests that it is possible to improve trajectory accuracy in healthy persons in conditions with a visuoproprioceptive conflict by reducing the activity of the somatosensory areas. This finding may be applied in the future to improving trajectory control when movements are monitored from an unfamiliar angle in a mirror or a video camera.

\section{References}

Bard C, Fleury M, Teasdale N, Paillard J, Nougier V (1995) Contribution of proprioception for calibrating and updating the motor space. Can J Physiol Pharmacol 73:246-254.

Boroojerdi B, Prager A, Muellbacher W, Cohen LG (2000) Reduction of human visual cortex excitability using 1-Hz transcranial magnetic stimulation. Neurology 54:1529-1531.

Chen R, Classen J, Gerloff C, Celnik P, Wassermann EM, Hallett M, Cohen LG (1997) Depression of motor cortex excitability by low-frequency transcranial magnetic stimulation. Neurology 48:1398-1403.

Corkin S, Milner B, Rasmussen T (1970) Somatosensory thresholds-contrasting effects of postcentral-gyrus and posterior parietal-lobe excisions. Arch Neurol 23:41-58.

Ghez C, Gordon J, Ghilardi MF (1995) Impairments of reaching movements in patients without proprioception. II. Effects of visual information on accuracy. J Neurophysiol 73:361-372.

Ghez C, Krakauer JW, Sainburg RL, Ghilardi M-F (2000) Spatial representations and internal models of limb dynamics in motor learning. In: The new cognitive neurosciences (Gazzaniga M, ed), pp 501-514. Cambridge, MA: MIT.

Guedon O, Gauthier G, Cole J, Vercher J-L, Blouin J (1998) Adaptation in visuomanual tracking depends on intact proprioception. J Mot Behav 30:234-248.

Harris JA, Miniussi C, Harris IM, Diamond ME (2002) Transient storage of a tactile memory trace in primary somatosensory cortex. J Neurosci 22:8720-8725.

Janwantanakul P, Magarey ME, Jones MA, Dansie BR (2001) Variation in shoulder position sense at mid and extreme range of motion. Arch Phys Med Rehabil 82:840-844.

Jeannerod M, Michel F, Prablanc C (1984) The control of hand movements in a case of hemianaesthesia following a parietal lesion. Brain 107:899-920.

Jones KE, Wessberg J, Vallbo A (2001) Proprioceptive feedback is reduced during adaptation to a visuomotor transformation: preliminary findings. NeuroReport 12:4029-4033.

Knecht S, Ellger T, Breitenstein C, Bernd Ringelstein E, Henningsen H 
(2003) Changing cortical excitability with low-frequency transcranial magnetic stimulation can induce sustained disruption of tactile perception. Biol Psychiatry 53:175-179.

Kosslyn SM, Pascual-Leone A, Felician O, Camposano S, Keenan JP, Thompson WL, Ganis G, Sukel KE, Alpert NM (1999) The role of area 17 in visual imagery: convergent evidence from PET and rTMS. Science 284:167-170.

Lajoie Y, Paillard J, Teasdale N, Bard C, Fleury M, Forget R, Lamarre Y (1992) Mirror drawing in a deafferented patient and normal subjects: visuoproprioceptive conflict. Neurology 42:1104-1106.

Lisanby SH, Gutman D, Luber B, Schroeder C, Sackeim HA (2001) Sham TMS: intracerebral measurement of the induced electrical field and the induction of motor-evoked potentials. Biol Psychiatry 49:460-463.

McKay DR, Ridding MC, Miles TS (2003) Magnetic stimulation of motor and somatosensory cortices suppresses perception of ulnar nerve stimuli. Int J Psychophysiol 48:25-33.

Milner B, Corkin S, Teuber HL (1968) Further analysis of the hippocampal amnesic syndrome: 14 year follow-up study of H.M. Neuropsychologia 6:215-234.

Naito E, Roland PE, Ehrsson HH (2002) I feel my hand moving: a new role of the primary motor cortex in somatic perception of limb movement. Neuron 36:979-988.

Nudo RJ, Friel KM, Delia SW (2000) Role of sensory deficits in motor impairments after injury to primary motor cortex. Neuropharmacology 39:733-742.

Paillard J, Brouchon M (1968) Active and passive movements in the callibration of position sense. In: The neuropsychology of spatially oriented behavior (Freedman SJ, ed), pp 37-55. Homewood, IL: Dorsey.

Pascual-Leone A, Bartres-Faz D, Keenan JP (1999) Transcranial magnetic stimulation: studying the brain-behaviour relationship by induction of "virtual lesions." Philos Trans R Soc Lond B Biol Sci 354:1229-1238.

Pause M, Kunesch E, Binkofski F, Freund HJ (1989) Sensorimotor disturbances in patients with lesions of the parietal cortex. Brain 112:1599-1625.
Poulton EC (1974) Tracking skill and manual control. New York: Academic.

Ragert P, Becker M, Tegenthoff M, Pleger B, Dinse HR (2004) Sustained increase of somatosensory cortex excitability by $5 \mathrm{~Hz}$ repetitive transcranial magnetic stimulation studied by paired median nerve stimulation in humans. Neurosci Lett 356:91-94.

Rothwell JC, Traub MM, Day BL, Obeso JA, Thomas PK, Marsden CD (1982) Manual motor performance in a deafferented man. Brain 105:515-542.

Rymer WZ, D'Almeida A (1980) Joint position sense: the effects of muscle contraction. Brain 103:1-22.

Sanes JN, Mauritz KH, Evarts EV, Dalakas MC, Chu A (1984) Motor deficits in patients with large-fiber sensory neuropathy. Proc Natl Acad Sci USA 81:979-982.

Sanes JN, Mauritz KH, Dalakas MC, Evarts EV (1985) Motor control in humans with large-fiber sensory neuropathy. Hum Neurobiol 4:101-114.

Semenza C (2001) Disorders of body representation. In: Handbook of neuropsychology, Vol 3, Ed 2 (Berndt RS, ed), pp 285-303. Amsterdam: Elsevier.

Siebner HR, Rothwell J (2003) Transcranial magnetic stimulation: new insights into representational cortical plasticity. Exp Brain Res 148:1-16.

Strick PL, Preston JB (1982) Two representations of the hand in area 4 of a primate. II. Somatosensory input organization. J Neurophysiol 48:150-159.

Sugishita M, Takayama Y (1993) Paraesthesia elicited by repetitive magnetic stimulation of the postcentral gyrus. NeuroReport 4:569-570.

Velay JL, Roll R, Paillard J (1989) Elbow position sense in man: contrasting results in matching and pointing. Hum Mov Sci 8:177-193.

Wassermann EM, Wang B, Zeffiro TA, Sadato N, Pascual-Leone A, Toro C, Hallett M (1996) Locating the motor cortex on the MRI with transcranial magnetic stimulation and PET. NeuroImage 3:1-9.

Wolpert DM, Goodbody SJ, Husain M (1998) Maintaining internal representations: the role of the human superior parietal lobe. Nat Neurosci $1: 529-533$. 